\title{
Sleeping Sickness Disrupts the Sleep-Regulating Adenosine System
}

\author{
${ }^{\circ}$ Filipa Rijo-Ferreira, ${ }^{1,2 *}{ }^{\circledR}$ Theresa E. Bjorness, ${ }^{3,4 *}{ }^{\circledR}$ Kimberly H. Cox,${ }^{1}$ Alex Sonneborn, ${ }^{1,3}$ \\ ${ }^{\circledR}$ Robert W. Greene, ${ }^{1,3}$ and ${ }^{\circledR}$ Joseph S. Takahashi ${ }^{1,2}$ \\ ${ }^{1}$ Department of Neuroscience, Peter O’Donnell Jr. Brain Institute, University of Texas Southwestern Medical Center, Dallas, Texas 75390-9111, \\ ${ }^{2}$ Howard Hughes Medical Institute, University of Texas Southwestern Medical Center, Dallas, Texas 75390-9111, ${ }^{3}$ Department of Psychiatry, \\ University of Texas Southwestern Medical Center, Dallas, Texas 75390-9111, and ${ }^{4}$ Research Service, VA North Texas Health Care System, Dallas, \\ Texas $75216-7167$
}

Patients with sleeping sickness, caused by the parasite Trypanosoma brucei, have disruptions in both sleep timing and sleep architecture. However, the underlying cause of these sleep disturbances is not well understood. Here, we assessed the sleep architecture of male mice infected with $T$. brucei and found that infected mice had drastically altered sleep patterns. Interestingly, T. brucei-infected mice also had a reduced homeostatic sleep response to sleep deprivation, a response modulated by the adenosine system. We found that infected mice had a reduced electrophysiological response to an adenosine receptor antagonist and increased adenosine receptor gene expression. Although the mechanism by which T. brucei infection causes these changes remains to be determined, our findings suggest that the symptoms of sleeping sickness may be because of alterations in homeostatic adenosine signaling.

Key words: adenosine; homeostasis; sleep; sleeping sickness

\section{Significance Statement}

Sleeping sickness is a fatal disease that disrupts the circadian clock, causes disordered temperature regulation, and induces sleep disturbance. To examine the neurologic effects of infection in the absence of other symptoms, in this study, we used a mouse model of sleeping sickness in which the acute infection was treated but brain infection remained. Using this model, we evaluated the effects of the sleeping sickness parasite, Trypanosoma brucei, on sleep patterns in mice, under both normal and sleep-deprived conditions. Our findings suggest that signaling of adenosine, a neuromodulator involved in mediating homeostatic sleep drive, may be reduced in infected mice.

\section{Introduction}

Sleeping sickness (human African trypanosomiasis) is a disease endemic to sub-Saharan Africa. It is caused by the parasite Trypanosoma brucei, which is transmitted to humans by the tsetse fly (Franco et al., 2014). Sleeping sickness occurs in two

\footnotetext{
Received Apr. 30, 2020; revised Sep. 28, 2020; accepted 0ct. 11, 2020

Author contributions: F.R.-F., T.E.B., and J.S.T. designed research; F.R.-F., T.E.B., and A.S. performed research; F.R.-F., T.E.B., K.H.C., A.S., and R.W.G. analyzed data; K.H.C. wrote the paper.

${ }^{*}$ F.R.-F. and T.E.B. contributed equally to the present study.

This work was supported by the National Institutes of Health (NIH) Grant NIGMS K99GM132557 (to F.R.-F.), the VA The Biomedical Laboratory Research \& Development Service CDA Grant IK2BX002531 (to T.E. B.), the NIH Grant R01MH080297 and World Premier International program for IIIS (to R.W.G.), and funding from Howard Hughes Medical Institute (J.S.T.). We thank Nelly Garduño and Iza Kornblum for their technical assistance and Fernando Augusto for artwork. J.S.T. is an Investigator and F.R.-F. is an Associate in the Howard Hughes Medical Institute. The views expressed in this article are those of the authors and do not necessarily reflect the position or policy of the Department of Veterans Affairs or the United States government.

The authors declare no competing financial interests.

Correspondence should be addressed to Joseph S. Takahashi at joseph.takahashi@utsouthwestern.edu.

https://doi.org/10.1523/JNEUROSCI.1046-20.2020

Copyright $\odot 2020$ the authors
}

phases. In the first, hemolymphatic phase, T. brucei parasites are primarily found in the bloodstream, interstitial spaces of peripheral organs, and lymphatic system (Caljon et al., 2016; Capewell et al., 2016; Trindade et al., 2016). The main symptoms during this first phase of the disease include fever, fatigue, and pain. In the second, meninge-encephalitic stage, the parasites invade the central nervous system and cause psychiatric symptoms as well as the progressive disruption of circadian rhythms, including altered hormonal secretion, body temperature rhythms, daytime somnolence, and nighttime insomnia (Buguet et al., 2001; Blum et al., 2006; Tesoriero et al., 2019).

In humans, sleep can be divided into four stages: three substages of non-rapid eye movement (NREM) sleep [stage 3-4 also called slow wave sleep (SWS)] and REM sleep. These stages can be delineated by differences in brain activity as measured by electroencephalography (EEG) and other physiological parameters, such as muscle and eye movements (Jafari and Mohsenin, 2010). In addition to changes in sleep timing, sleeping sickness patients have altered sleep architecture, with REM episodes occurring immediately after sleep onset [sleep-onset REM (SOREM)] instead 
of after NREM (Buguet et al., 2001, 2005). This change in sleep structure is similar to what is seen with narcolepsy (Nishino and Mignot, 1997; Fujiki et al., 2009). Interestingly, while neither sleeping sickness nor narcolepsy causes increased total sleep across the 24-h period (Dantz et al., 1994; Buguet et al., 2001), narcoleptic patients do not have altered circadian rhythms (Dantz et al., 1994). T. brucei is also distinct from other infections, which tend to increase sleep (Krueger and Opp, 2016; Tesoriero et al., 2019). How the T. brucei parasite produces these specific alterations to the sleep system remains an open question (Lundkvist et al., 2004; Rijo-Ferreira and Takahashi, 2020).

Sleep is regulated by interactions of the circadian system with a complex homeostatic network that balances sleep need with arousal (Borbély et al., 2016). Although there are many signals involved in maintaining arousal, an increase in extracellular adenosine during wakefulness is thought to be a primary driver of both homeostatic sleep need (Porkka-Heiskanen et al., 1997; Saper et al., 2005; Bjorness et al., 2016; Bringmann, 2018) and motivation-related arousal (Lazarus et al., 2019). In addition, allostatic mechanisms, such as the production of somnogens during infection, also promote sleep (Bringmann, 2018; Toda et al., 2019). We previously showed that T. brucei infection shortens the period of circadian behavioral activity in mice and disrupts the molecular circadian clock within the suprachiasmatic nucleus (SCN) of the hypothalamus (Rijo-Ferreira et al., 2018). However, the effects of $T$. brucei on homeostatic drivers of sleep are unclear.

The purpose of the present study was to examine the effects of $T$. brucei infection on sleep architecture in a mouse model of sleeping sickness. We found that, similarly to the human infection, T. brucei-infected mice had altered sleep architecture. Interestingly, infection reduced the ability of mice to achieve or maintain REM sleep. In addition, infected mice lacked a response to sleep deprivation, which may indicate a change in their ability to achieve sleep or reduced sleep drive in the face of homeostatic challenge. Electrophysiological experiments and quantification of gene expression suggested that these behavioral changes were not due solely to inflammation induced by infection, but rather may be caused by altered homeostatic adenosine signaling.

\section{Materials and Methods}

Ethics statement

All animal care and experimental procedures were performed in accordance with University of Texas Southwestern Medical Center (UTSW) IACUC guidelines, approved by the Ethical Review Committee at UTSW.

\section{Housing and surgical procedures}

Adult (8- to 12-week-old) male C57BL/6J mice from the UTSW Mouse Breeding Core Facility were housed in standard mouse cages with food and water provided ad libitum. Cages were in a temperature-controlled environment in a T24 12/12 h light/dark cycle. To determine sleep/waking activity, electroencephalogram (EEG) and Electromyography (EMG) electrodes were surgically implanted as described previously (Bjorness et al., 2009). Briefly, mice were anesthetized with isoflurane and the scalp was sheared and cleaned. Mice were then placed in a stereotax (Kopf) and four holes were drilled in the skull. Two EEG electrodes (Plastics One) were placed bilaterally over the frontal cortex (AP +1.7 mm, ML $\pm 1.77 \mathrm{~mm}$ ) and two electrodes were placed bilaterally over the parietal cortex (AP $-1.7 \mathrm{~mm}, \mathrm{ML} \pm 2 \mathrm{~mm}$ ). Two EMG electrodes (Plastics One) were placed bilaterally into the nuchal musculature. Electrode pins were threaded into a six-pin pedestal and affixed to the skull with dental adhesive (Fisher). After the surgery, the implant base was coated with antibiotic and animals were given Buprenex $(0.1 \mathrm{ml}$, i.p.) for analgesia.
Animals were given $14 \mathrm{~d}$ to recover from surgery before tether adaption and were housed singly following surgery.

\section{Infection and suramin treatment}

Infections were performed by intraperitoneal injection of $2000 \mathrm{~T}$. brucei brucei AnTat $1.1^{\mathrm{E}}$ parasites between Zeitgeber time (ZT)9 and ZT12 (3-h interval before lights are off; Rijo-Ferreira et al., 2018). This is a T. brucei AnTat $1.1^{\mathrm{E}} 90-13$, pleomorphic clone, from a transgenic cell-line encoding the tetracycline repressor and T7 RNA polymerase (Engstler and Boshart, 2004). For all injections, the parasite cryostabilates used were obtained from a previous $5-\mathrm{d}$ infection. Before infection, T. brucei cryostabilates were thawed and parasite viability and numbers were assessed by mobility under a microscope. Dilutions of parasite densities were performed in HMI-11 medium to an inoculum of $100 \mu \mathrm{l}$. Parasitemia was assessed by tail vein blood collected into HMI-11 medium and assessed with a Neubauer chamber under the microscope. On day 21 postinfection, at ZT9-ZT12, suramin $(20 \mathrm{mg} / \mathrm{kg}$, in $100 \mu \mathrm{l})$ was administered intraperitoneally to both infected and uninfected control mice.

\section{Experimental groups}

Three cohorts of controls and trypanosome-infected mice were used (Fig. 1A, groups 1-3). The first cohort ( $n=4$ /group) underwent undisturbed (baseline) EEG/EMG recording before and following infection (details for this design below, EEG/EMG recording). The second cohort ( $n=6$ /group) underwent baseline recording before infection, a second baseline following infection, and a recording after sleep deprivation (details for this design below, Sleep deprivation protocol). The third cohort ( $n=12$ controls, $n=15$ trypanosome infected) underwent infection followed by tissue collection for in vitro electrophysiology [details for this design below, Hippocampal field recordings (paired-pulse facilitation; PPF)] or gene expression measurement (details for this design below, Quantitative real-time PCR).

\section{EEG/EMG recordings}

One cohort of control $(n=4)$ and trypanosome-infected $(n=4)$ mice were monitored continuously for $48 \mathrm{~h}$ both preinfection (baseline) and $85 \mathrm{~d}$ postinfection (Fig. $1 A$, group 1). For recordings, cages were placed in custom wooden boxes and a cable tether was secured to the implant and a commutator (Plastics One) located on the lid of the box. Animals were given at least $7 \mathrm{~d}$ to acclimate to the cable before baseline recordings. Animals were disconnected from the tether between recordings and returned to the colony room.

EEG and EMG activity were recorded using a Grass model 15LT amplifier system (Grass Technologies) with a sampling frequency of $256 \mathrm{~Hz}$ and filtered between 0.1 and $300 \mathrm{~Hz}$. Data were analyzed using a custom MATLAB-based sleep scorer program. EEG and EMG signals were autosorted in 10-s epochs followed by manual check of state assignment and flagging of artifacts. Epochs with artifacts were excluded from all power analyses (15,679 out of 681,070 epochs were excluded, or $2.3 \%$ ). Standard state criteria were used: waking was characterized by low amplitude, high-frequency EEG activity and high EMG activity, SWS by high-amplitude, low-frequency EEG activity with negligible EMG modulation, and REM sleep by low amplitude EEG activity with occasional muscle twitches and low overall EMG activity. As is typical for rodent models, SWS was not distinguished from NREM. $\delta$ Power was defined as EEG slow wave activity (SWA) power within a bandwidth $0.5-4.5 \mathrm{~Hz}$ and normalized to average $\gamma$ power $(30-50 \mathrm{~Hz})$ in which $\gamma$ was averaged across all artifact-free epochs across states and across the entire baseline period, unless otherwise noted. Episodes began with 30 consecutive seconds of a single state and ended with 30 consecutive seconds of a single different state. State transitions shorter than three epochs (30 s) were ignored.

Sleep deprivation protocol

A second cohort of animals ( $n=6 /$ group) underwent surgery, baseline recording, infection, and suramin treatment followed by sleep deprivation (Fig. 1A, group 2) Mice were placed into bottomless cages suspended above a treadmill, with the cable tether attached to a 
A
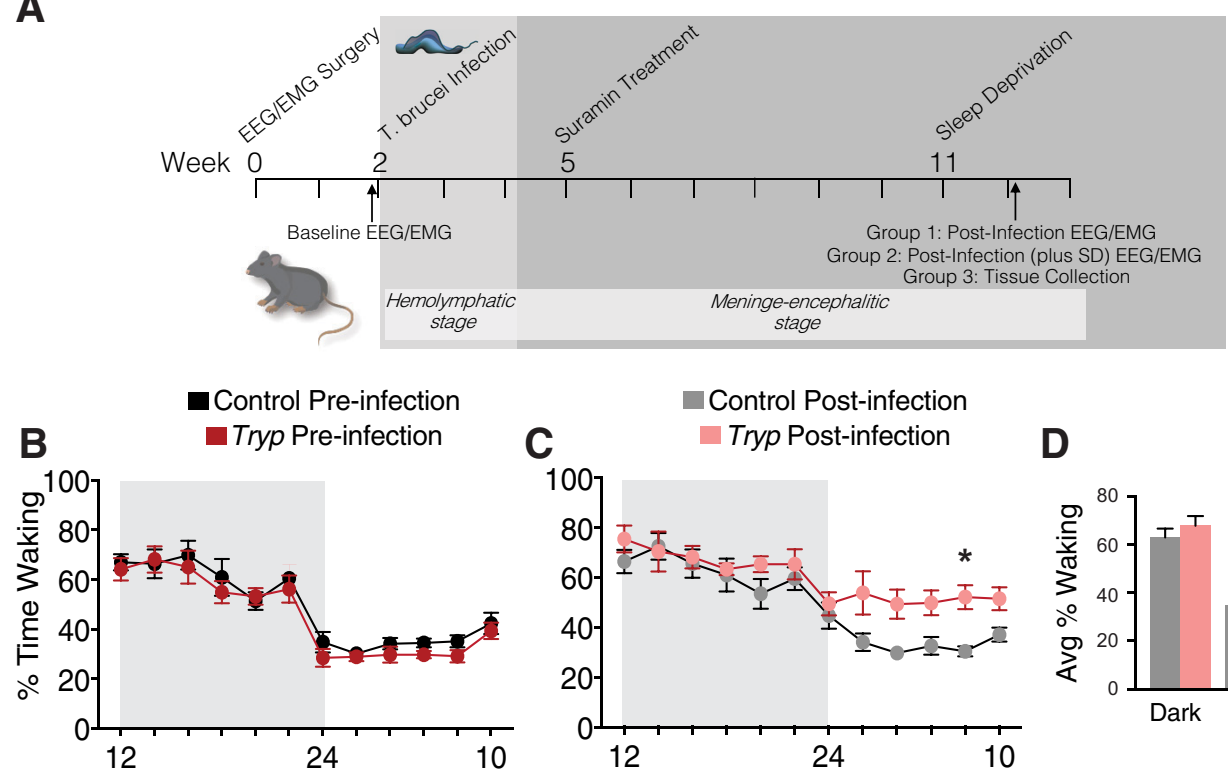

D

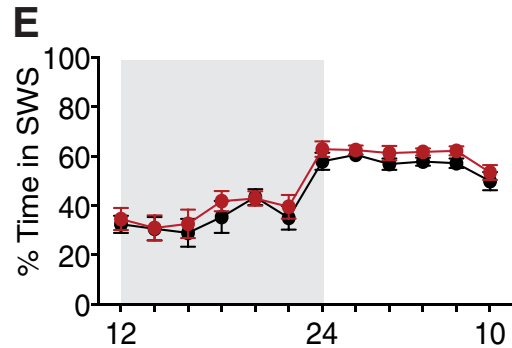

$\mathbf{F}$
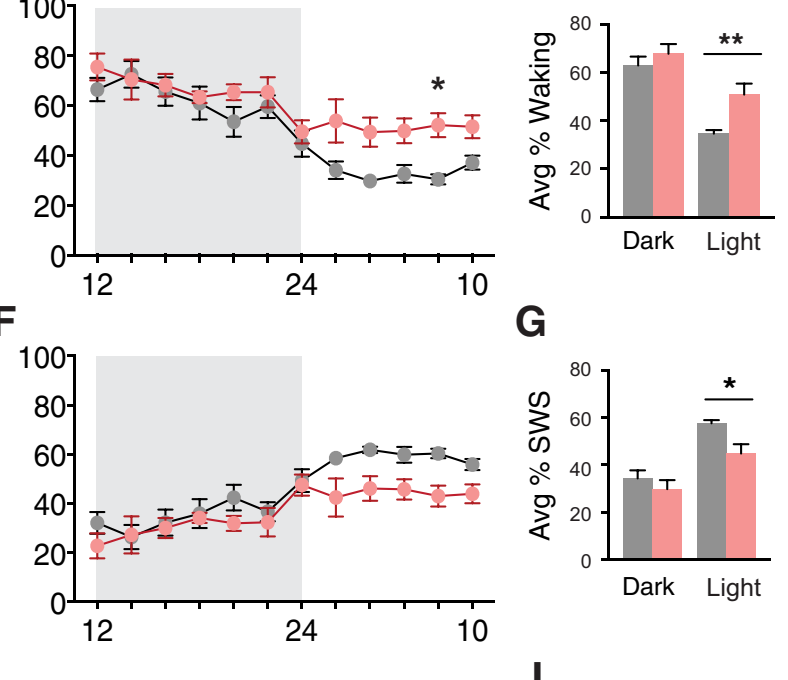

G
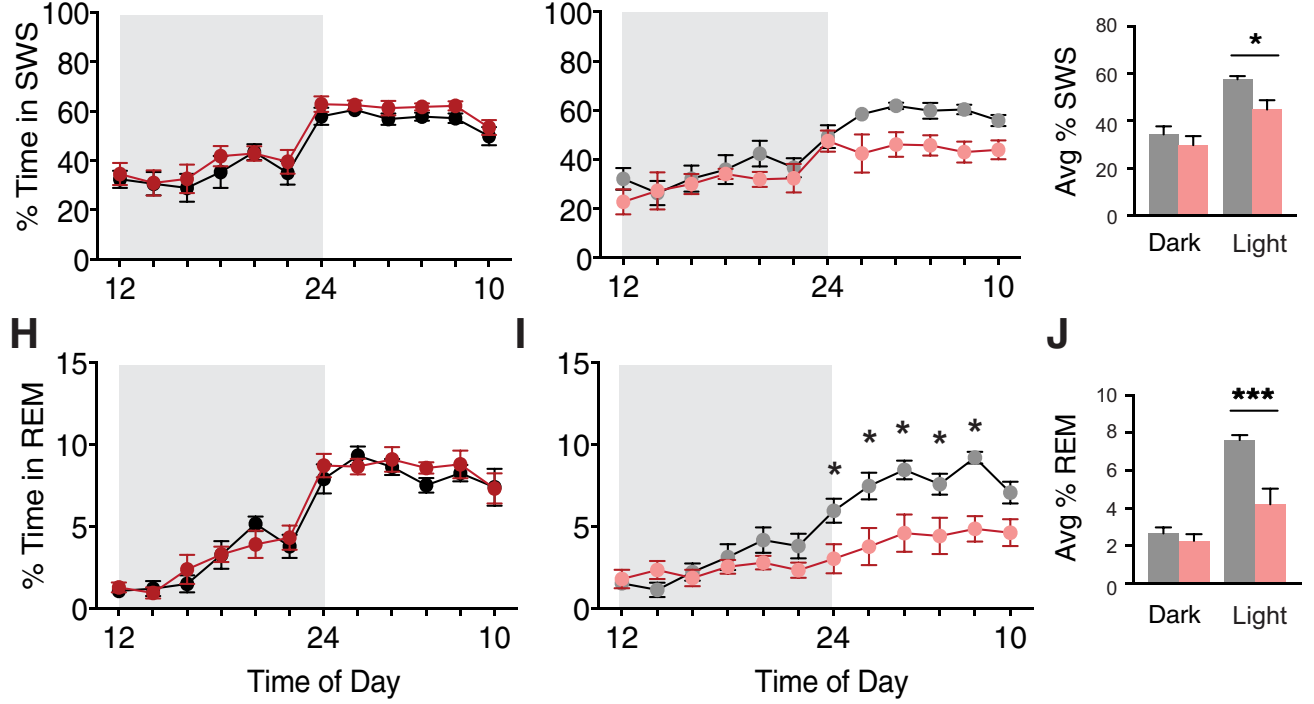

Figure 1. T. brucei infection changes sleep architecture in mice. $A$, Timeline of methods for all experiments. In the first experiment, mice $(n=4 /$ group) were evaluated via EEG and EMG preinfection and $85 \mathrm{~d}$ postinfection with T. brucei. In the second experiment, mice ( $n=6 /$ group) were evaluated via EEG and EMG preinfection and starting at 76-83 d postinfection (baseline and SD). $\boldsymbol{B}$, Before infection, there were no differences between groups in the \% of total time spent waking. C, Postinfection, mice spent significantly more time waking (two-way ANOVA; main effects of time $F_{(11,187)}=17.78, p<0.0001$; and group $F_{(1,17)}=6.128, p=0.0241 ; *$ denotes group difference after multiple corrections). $\boldsymbol{D}$, When \% time waking was averaged over the dark and light periods, infected animals spent significantly more time waking in the light phase (one-way ANOVA; $F_{(3,34)}=2.137, * * p=0.0054$ ). $E$, Before infection, there were no differences between groups in the \% of total time spent in SWS. $\boldsymbol{F}$, Postinfection, mice tended to spend less time in SWS. G, However, this difference was only statistically significant when averaged over the dark and light periods (one-way ANOVA; $F_{(3,34)}=2.106, * p=0.0162$ ). $\boldsymbol{H}$, Before infection, there were no differences between groups in the $\%$ total time spent in REM sleep. $I$, Postinfection, mice spent significantly less time in REM sleep (two-way ANOVA; main effects of time $F_{(11,187)}=21.00, p<0.0001$; and group $F_{(1,17)}=11.32, p=0.0037$; and an interaction $F_{(11,187)}=4.340, p<0.0001 ; *$ denotes group differences after multiple corrections). J, When \% time in REM sleep was averaged over the dark and light periods, infected animals spent significantly more time in REM sleep in the light (daytime; Brown-Forsythe ANOVA; $F_{(3,15.7)}=25.60, * * * p=0.0061$ ).

counterbalanced lever arm (Instech Laboratories) to facilitate free movement. Animals were given $7 \mathrm{~d}$ to acclimate to the recording tether and treadmill environment before the initial recordings. Following infection, mice underwent 24-h baseline recording, followed by sleep deprivation with baseline occurring between one time, $76-83 \mathrm{~d}$ postinfection. The sleep deprivation protocol varied across animals since the infected mice were unable to maintain the necessary constant motion of the planned chronic, partial sleep deprivation protocol previously used to assess sleep homeostasis in adenosine-modulated animals (Bjorness et al., 2016). Two control and two infected mice underwent 4-h sleep deprivation followed by 2 -h recovery sleep repeated eight consecutive times; both controls survived while both infected mice died. Next, one control and one infected mouse underwent 4 -h sleep deprivation with $2 \mathrm{~h}$ of recovery during the light phase only (i.e., eight total hours of sleep deprivation per day); again, the control mouse survived, and the infected mouse died.

For the remaining mice ( $n=3$ control, $n=3$ infected), sleep deprivation occurred via a combination of gentle handling and treadmill movement. For control mice, the experimenter would turn on the treadmill for short periods when a mouse would appear to fall asleep. Infected mice were placed into a cage during periods of constant treadmill movement and waking was enforced by gentle handling procedures such as tapping the side of the cage or swirling the bedding within the cage. Mice underwent two bouts of 4-h sleep deprivation followed by $2 \mathrm{~h}$ of recovery sleep repeated twice during the light phase of $1 \mathrm{~d}$. All mice survived this sleep deprivation procedure; however, one infected mouse was 
Table 1. Primer sequences for qRT-PCR

\begin{tabular}{ll}
\hline Adk & F: AATTTGGAAGCGTGGTGTG \\
Adora1 & R: CCCCAAGTCCATCAGATGTC \\
& F: AGAACCACCTCCACCCTTCT \\
Ifn $\gamma$ & R: TACTCTGGGTGGTGGTCACA \\
$\| 10$ & F: CACACTGCATCTTGGCTTTG \\
& R: TCTGGCTCTGCAGGATTTTC \\
$\| 1 \beta$ & F: CTCATGGGTCTGGGAAGAG \\
& R: CATTCCCAGAGGAATTGCAT \\
Tnf $\alpha$ & F: GCCCATCCTCTGTGACTCAT \\
& R: AGGCACAGGTATTTGTCG \\
& F: AATGGCCTCCTCTCATCAGTT \\
& R: CCACTTGGTGGTTGCTACGA \\
\hline
\end{tabular}

excluded from analysis because of poor signal quality (high percentage of epochs with artifact). With gentle handling, a high degree of sleep deprivation was maintained ( $>97 \%$ waking), though a small amount of total sleep was achieved ( 4 and $1 \mathrm{~min}$ in two controls and 2.83 and $3.17 \mathrm{~min}$ in two infected mice).

Hippocampal field recordings (paired-pulse facilitation; $P P F$ )

Infected and control mice ( $n=8$ /group; Fig. $1 A$, group 3 ) were treated with suramin on day 21 postinfection, and $85 \mathrm{~d}$ postinjection mice were anaesthetized under isoflurane, and the brains removed and blocked following rapid decapitation. Coronal slices ( $300 \mu \mathrm{m}$ thick) containing the hippocampus were prepared using a vibratome (VT 1000S, Leica Microsystems) in ice cold $N$-methyl-D-glucosamine (NMDG) ringer solution then transferred to artificial CSF (aCSF) as described previously (Takeuchi et al., 2016). One slice per animal was used for ex vivo field recordings. Slices were transferred to a submersion chamber and were perfused with aCSF at a rate of $\sim 2 \mathrm{ml} \mathrm{min}^{-1}$ at $29-31^{\circ} \mathrm{C}$. Field recordings from the stratum radiatum of dorsal CA1 were acquired using a borosilicate glass electrode (1-3 M $\Omega$ ) filled with aCSF. A bipolar stimulating electrode was also placed in the stratum radiatum of CA1 within $100-200 \mu \mathrm{m}$ of the recording electrode and stimulation (one stimulus every $30 \mathrm{~s}$ ) was set to elicit a field EPSP (fEPSP) slope that was $~ 50 \%$ of the maximum value. A stable 15-min baseline was obtained, followed by paired pulse electrical stimulation using inter-pulse interval times of 10, 20 , 50, and $100 \mathrm{~ms}$, with six recordings per interval, averaged. An adenosine A1 receptor antagonist, 8-cyclopentyltheophylline (CPT) was then applied to the bath $(10 \mathrm{uM})$ for $15 \mathrm{~min}$ and the recording procedure was repeated. Data were acquired using P-Clamp 10.

\section{Quantitative real-time PCR}

Infected and control mice were treated with suramin (Fig. 1A, group 3); $85 \mathrm{~d}$ postinjection, at ZT5-ZT6, mice were euthanized with $\mathrm{CO}_{2}$. Blood $(200 \mu \mathrm{l})$ was collected via cardiac puncture and brain regions were dissected. Total RNA was extracted using TRIzol (tissue) and TRIzol LS (blood) following manufacturer's protocol (Thermo Fisher Scientific). cDNA was prepared using TaqMan Reverse Transcription Reagents (Thermo Fisher Scientific) with random hexamers. qRT-PCR was performed with Sybr Green in an Applied Biosystems 7500 Real-Time PCR System (RRID:SCR_018051) and normalized to Gapdh as a housekeeping gene. Primer sequences are listed in Table 1.

Experimental design and statistical analyses

Statistical analyses were performed using GraphPad Prism version 7.01 for Windows or 8.0.0 for Mac (GraphPad Software, RRID:SCR_002798). For time in state comparisons, data from cohorts 1 and 2 were averaged in 2-h bins and compared using two-way ANOVA (time $\times$ group) and then averaged within light and dark phases and compared using oneway ANOVA with Sidak's multiple comparisons test (within phase across group), or Brown-Forsythe tests when standard deviations were different between groups. REM was divided by total sleep (\%REM+\% NREM) in each 2-h bin and then averaged within light and dark phases and compared using one-way ANOVA with Sidak's multiple comparisons test. For episode number and duration, data were averaged within the light and dark phases and compared using one-way ANOVA with
Sidak's multiple comparison test. Data were then divided into bins based on duration (histogram) and compared using a two-way ANOVA with Sidak's multiple comparisons test (cumulative histogram for SWS duration) or Tukey's multiple comparisons test (histogram for REM duration). For REM histogram analysis, the episode duration criteria (30 s) were relaxed such that the distribution of shorter REM episodes (10-20 s) could be seen. State transitions were compared by calculating the number of sleep episodes that preceded different sleep episodes (SWS to REM and REM to SWS) and dividing by the total number of sleep specific episodes (i.e., [SWS to REM]/total SWS; [REM to SWS]/total REM). SWS transitions and REM transitions were compared between groups using a $t$ test.

Normalized SWS SWA power was averaged in 2-h bins and compared using two-way ANOVA (time by group), while average rebound was calculated by the percent change in SWS-SWA from baseline conditions to recovery conditions using time-matched circadian bins (i.e., ZT4-ZT6, ZT10-ZT12, ZT16-ZT18,, ZT22-ZT24) and compared using a $t$ test. Area under the curve (AUC) was calculated and compared between groups using a $t$ test.

SWA decrease across the light phase was calculated based on previously described methods (Nelson et al., 2013). For each baseline day, raw SWA in each epoch was normalized to the 24-h average SWA and the percentage change between the first and last consolidated 1-h sleep period, defined as a high proportion of sleep epochs within a 1-h bin, around the start and end of the light phase was calculated, after which the percent change was averaged across baseline days and compared via one-way ANOVA with Sidak's multiple comparison test (across group and time) and a Mann-Whitney $t$ test (between groups postinjection). Spectral density was determined during baseline conditions; individual 10 -s epochs were separated by state (waking, SWS, REM) after which raw spectral power in each $1-\mathrm{Hz}$ bin up to $100 \mathrm{~Hz}$ was normalized by the total power for that same state; the slowest $1-\mathrm{Hz}$ bin was excluded from the total power calculation. Fractional power was then averaged across epochs within each $1-\mathrm{Hz}$ bin to get an average spectral distribution from 2 to $100 \mathrm{~Hz}$ by state and compared across conditions using a two-way ANOVA (group by frequency). For clarity, Figure 2C,F,I are truncated at $15 \mathrm{~Hz}$; there were no significant differences above $15 \mathrm{~Hz}$.

Sleep latency under sleep deprived conditions was calculated as the waking duration from the beginning of sleep deprivation to the initial SWS episode during recovery (for gentle handling, first sleep occurred toward the end of the deprivation period) and was averaged across deprivations so that each mouse contributed one average sleep latency. A $t$ test was used for group comparisons. SOREMs are defined as direct transitions from waking to REM. All scored files were analyzed for transitions from waking episodes to REM episodes, but no such SOREMs were found. We also looked for epochs with continuous waking activity of at least $30 \mathrm{~s}$ followed by REM (Chen et al., 2006; Seke Etet et al., 2012), but again no SOREMs were identified.

For electrophysiological recordings, calculated paired-pulse ratios were compared via two-way repeated-measures ANOVA. qRT-PCR data were analyzed using the DDCT method and compared using unpaired $t$ tests with Welch's correction.

\section{Results}

\section{Altered sleep architecture in a mouse model of sleeping sickness}

To model the second, meninge-encephalitic stage of the human sleeping sickness infection (when sleep disturbances occur in the absence of acute symptomology), mice were infected with T. brucei then treated $21 \mathrm{~d}$ postinfection with suramin $(20 \mathrm{mg} / \mathrm{kg})$, which kills the parasite in peripheral tissues while allowing it to survive in neural tissues (Rijo-Ferreira et al., 2018). EEG and EMG recordings were used to assess sleep behavior (divided into three stages: waking, SWS, and REM sleep) in mice preinfection and $85 \mathrm{~d}$ postinfection (Fig. 1A). Uninfected, suramin-treated controls were also assessed at the same timepoints. Before infection, there were no differences between groups in the $\%$ of total 
time spent waking, in SWS, or in REM (Fig. $1 B, E, H)$. However, postinfection, mice spent significantly more time waking (two-way ANOVA; main effects of time $F_{(11,187)}=17.78, p<0.0001$; and group $F_{(1,17)}=6.128, p=0.0241$; Fig. $1 C$ ), and less time in REM sleep (two-way ANOVA; main effects of time $F_{(11,187)}=$ $21.00, p<0.0001$; and group $F_{(1,17)}=$ 11.32, $p=0.0037$; and an interaction $F_{(11,187)}=4.340, p<0.0001$; Fig. $\left.1 I\right)$. When activity was averaged over the light or dark, it was apparent that infected mice spent significantly more time in waking (one-way ANOVA; $F_{(3,34)}=$ 2.137, $* * p=0.0054$; Fig. $1 D)$, and less time in SWS (one-way ANOVA; $F_{(3,34)}=$ $2.106, * p=0.0162$; Fig. $1 G)$ or REM (Brown-Forsythe ANOVA; $F_{(3,15.7)}=$ $25.60, * * * p=0.0061$; Fig. $1 J)$ during the light phase. There was also a significant decrease in the REM to total sleep (REM+SWS) ratio during the light phase postinfection (Brown-Forsythe ANOVA; $\left.F_{(3,34)}=3.878, * p=0.0173\right)$. These findings are consistent with our previous report of infected mice shifting their daily activity profiles to become more diurnal, showing increased activity during the light (daytime), when nocturnal mice are usually resting (Rijo-Ferreira et al., 2018).

In order to assess whether sleep architecture was similar between infected and control mice, we also compared the distribution of sleep episodes (number and duration). While there were no differences in the number or duration of SWS episodes between groups before T. brucei infection, there was a leftward shift in the distribution of episode duration following infection (Fig. 2A,B); two-way ANOVA; main effects of time $F_{(65,1105)}=802.6, p<$ 0.0001 ; and group $F_{(1,17)}=9.697, p=$ 0.0063 ; and an interaction $F_{(65,1105)}=11.35$, $p<0.0001$ ), indicative of more SWS episodes of shorter duration. These findings are consistent with sleep episode fragmentation that is associated with a loss of adenosine signaling (Bjorness et al., 2016). As with SWS, there were no differences in the number or duration of REM episodes before infection. However, postinfection, mice had fewer 10, 20, 60, and 90-s REM episodes as compared with controls (Fig. 2C, $D)$; two-way ANOVA; main effects of time $F_{(11,187)}=29.06$, $p<0.0001$; and group $F_{(1,17)}=15.93, p=0.0009$; and an interaction $\left.F_{(11,187)}=3.512\right)$, while total REM duration was unchanged. Example hypnograms of sleep/waking state across a 24-h period (starting at lights off) are shown in Figure 2E,F. In addition to changes in sleep state composition between groups, T. brucei also influenced transitions between sleep/waking states with a lower percentage of SWS episodes followed by REM episodes ( $t$ test; $\left.t_{(17)}=4.021 ; p=0.0009\right)$ and REM episodes followed by SWS episodes in infected mice $\left(t\right.$ test; $t_{(17)}=2.076 ; p=0.0534$; examples of
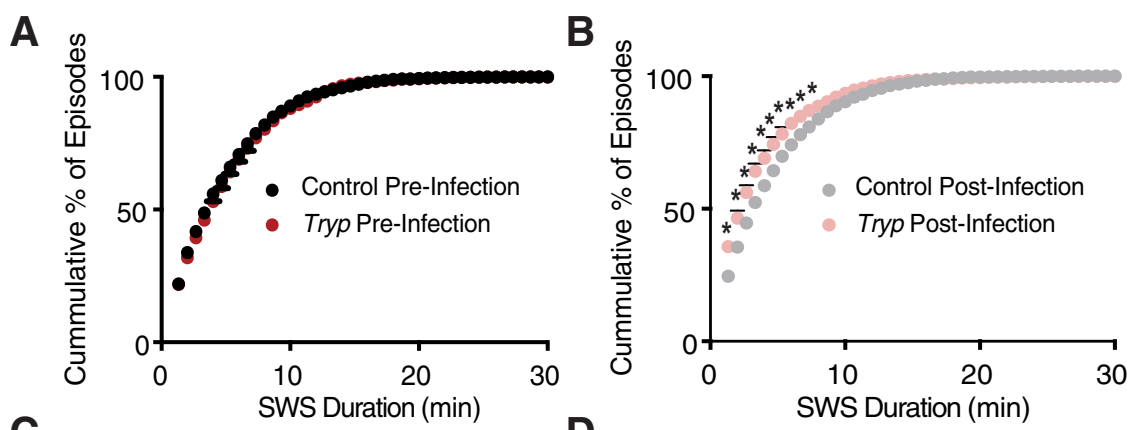

E

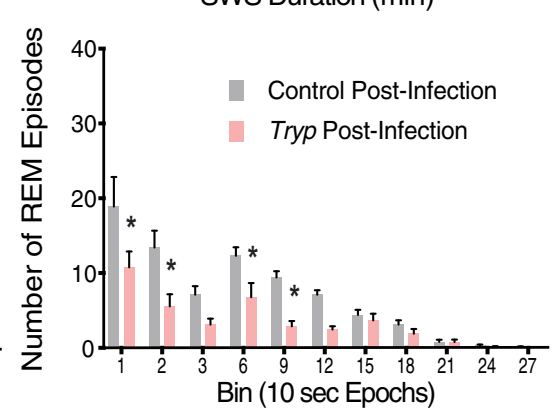

Figure 2. T. brucei infection reduces the duration of SWS and REM episodes. $\boldsymbol{A}$, When SWS episodes were calculated by cumulative duration across both the dark and light stages, there were no differences between groups preinfection. $\boldsymbol{B}$, In contrast, postinfection mice clearly had more SWS episodes of short duration than the controls (two-way ANOVA; main effects of time $F_{(65,1105)}=802.6, p<0.0001$; and group $F_{(1,17)}=9.697, p=0.0063$; and an interaction $F_{(65,1105)}=11.35$, $p<0.0001$. C, When REM episodes were binned by duration (10-s epochs) across both the dark and light phases, there were no differences between groups preinfection. $\boldsymbol{D}$, However, postinfection, mice had fewer 10-, 20-, 60-, and 90-s REM interaction $F_{(11,187)}=3.512, p=0.0002$; * denotes group differences after multiple corrections). $\boldsymbol{E}, \boldsymbol{F}$, Example hynograms showing the time course of wake, SWS, and REM across a 24-h period for control $(\boldsymbol{E})$ and infected $(\boldsymbol{F})$ mice. The yellow bar indicates lights on. Open circles indicate REM to waking transitions, closed circles indicate REM to SWS transitions.

REM to waking transitions and REM to SWS transitions are indicated in Fig. 2E,F). This higher percentage of SWS and REM episodes preceding waking indicates sleep fragmentation following infection. Taken together, these results indicate that T. brucei infection not only alters the circadian system (i.e., when animals are sleeping), but also likely affects the other systems regulating sleep homeostasis (Borbély et al., 2016).

To investigate the changes to sleep patterns further, we focused on the light phase, the predominant sleep period of mice where there were differences in the time spent in each phase of sleep (Fig. $1 D, G, J)$. There were no differences between groups in the total number of waking episodes or waking duration (Fig. $3 A, B)$. However, following infection, mice showed a shift toward 
—Control Pre-infection $\square$ Tryp Pre-infection $\square$ Control Post-infection $\square$ Tryp Post-infection

A

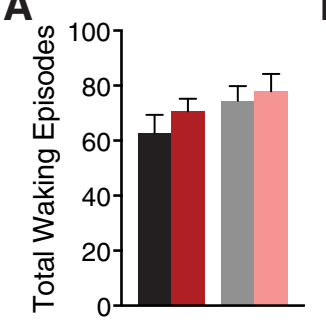

D

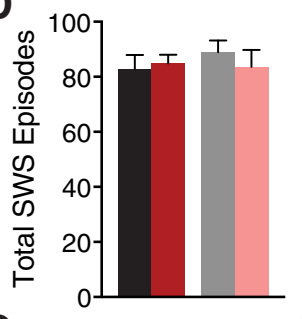

G

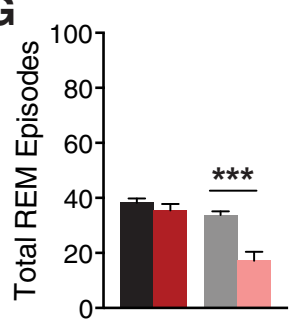

$B$

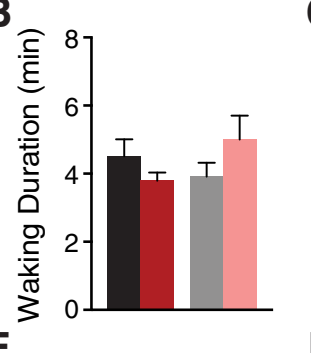

$\mathrm{E}$

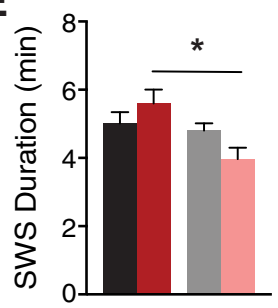

$\mathrm{H}$

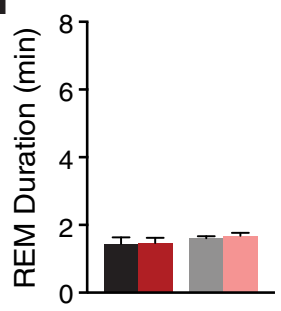

C

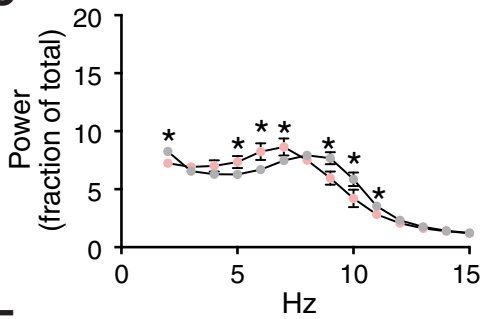

$\mathbf{F}$

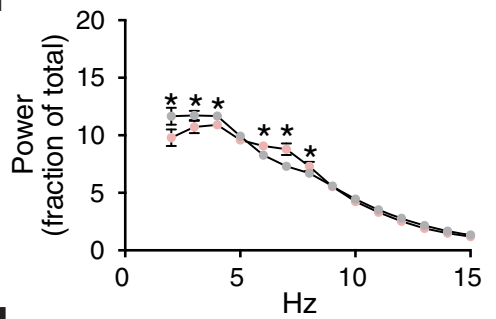

I

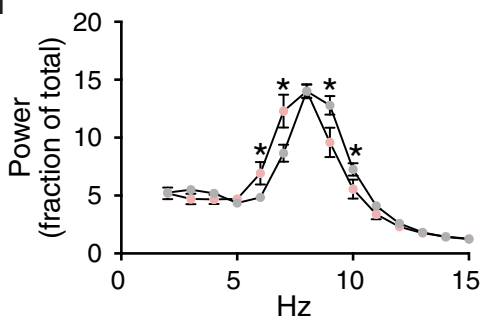

Figure 3. T. brucei infection reduces the number of REM episodes and the duration of SWS. In the light phase, there were no effects of infection on the total number $(\boldsymbol{A})$ or duration $(\boldsymbol{B})$ of waking episodes. $\boldsymbol{C}$, Following infection, mice showed a shift toward lower frequency activity during waking (two-way ANOVA; interaction of time and group $F_{(98,882)}=3.188$, $p<0.0001)$. $\boldsymbol{D}$, There was also no effect of infection on the total number of SWS episodes, $(\boldsymbol{E})$ but infection reduced the duration of SWS (one-way ANOVA, $F_{(3,34)}=4.119, * p=0.0088$ ) and $(\boldsymbol{F})$ shifted activity toward higher frequencies during SWS (two-way ANOVA; interaction of time and group $\left.F_{(98,882)}=3.322, p<0.0001\right)$. $\mathbf{G}$, Infected mice also displayed a reduced number of REM episodes (one-way ANOVA, $F_{(3,34)}=18.43$, ***p $<0.0001$ ), but $(\boldsymbol{H})$ there were no effects of infection on total REM duration, (I) while in infected animals activity was shifted to lower lower frequency during REM (interaction of time and group $\left.F_{(98,882)}=3.912, p<0.0001\right)$. There were no effects of infection on any of these measures in the dark; $*$ denotes group differences after multiple corrections.

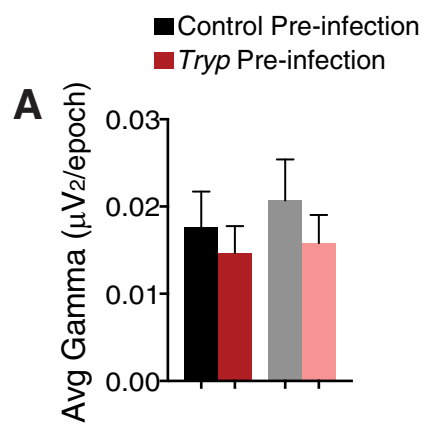

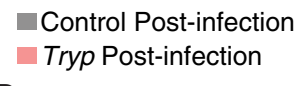

B

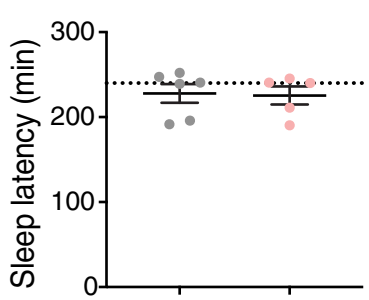

Figure 4. T. brucei infection had no effect on $\gamma$ power or sleep latency. $\boldsymbol{A}$, There were no differences in $\gamma$ power $(30-50 \mathrm{~Hz})$ preinfection or postinfection. $\boldsymbol{B}$, There were no differences between control and infected animals in their sleep latency under sleep deprivation conditions. The dashed line indicates the end of the 4-h deprivation period in which the treadmill belt stopped or gentle handling ceased. A subset of animals in each group achieved sleep during the deprivation period under the gentle handling method.

slower spectral frequency activity during waking (two-way ANOVA; interaction of time and group $F_{(98,882)}=3.188$, $p<0.0001$; Fig. $3 C$ ), consistent with the idea that infected mice spend more time in quiet wakefulness during the light phase. Infection also decreased the average duration of SWS episodes

(one-way ANOVA, $F_{(3,34)}=4.119$; Fig. $3 E$ ) with a shift toward higher spectral frequency in SWS (two-way ANOVA; interaction of time and group $F_{(98,882)}=3.322$, $p<0.0001$; Fig. $3 F$ ). The average number of REM episodes was also decreased (oneway ANOVA, $F_{(3,34)}=18.43$; Fig. $\left.3 G\right)$ with a shift toward lower spectral frequency activity during REM (interaction of time and group $F_{(98,882)}=3.912$, $p<0.0001$; Fig. $3 I$ ), suggesting a reduced ability to enter REM. Together, the decrease in the number of REM episodes and the reduction in SWS duration after infection suggested that $T$. brucei infection may impede REM generation. Although the disruption of sleep architecture is similar to what has been described for sleeping sickness patients, we were not able to detect direct transitions from waking to REM (SOREM) episodes in T. brucei-infected mice, which is different from what has been shown in T. brucei gambiense-infected or T. brucei rhodesienseinfected humans (Buguet et al., 1993) and T. brucei brucei-infected rats (Laperchia et al., 2016).

\section{T. brucei infection reduces homeostatic sleep rebound in mice}

In order to evaluate the quality of SWS after $T$. brucei infection, we quantified SWA during SWS across the entire measurement period. High-frequency EEG waves present during wakefulness (i.e., $\gamma$ power) did not differ between groups (Fig. 4A); therefore, this parameter was used to normalize SWS SWA power (Bjorness et al., 2016). As expected, before infection, all mice had peak SWA during the dark phase, indicative of increasing sleep drive which develops with extended periods of wakefulness (in nocturnal animals at night). However, after infection, the peak of SWA during SWS was flattened over the circadian cycle, hinting at overall circadian disruption and a reduction in sleep drive in infected mice (Fig. $5 A, B)$. AUC analysis for SWS SWA indeed showed that the infected group had a reduced SWS SWA area as compared with controls ( $t$ test; $t_{(192)}=2.003$; $p=0.0466$; Fig. $5 C$ ).

A decrease in SWA during SWS is an indicator of an overall decrease in sleep drive as the animal rests. Examples of SWA decreases across the light phase are shown for a control (Fig. 5D) and infected (Fig. 5E) mouse. When we calculated the percent decrease in SWA from a consolidated sleep period near the beginning of the light phase to a consolidated sleep period near the end of the light phase, the infected mice had less of a decrease than controls $\left[t\right.$ test; $t_{(18)}=1.962 ; \# p=0.0654$ (Fig. $5 F$ ); examples shown in Fig. 3D,E show $25.5 \%$ and $12 \%$ decrease, respectively]. These findings may indicate that infected mice do not have a normal accumulation of sleep need during wakefulness, i.e., they start out with a decreased drive, either because of a change in rate of sleep need accumulation (Franken et al., 2001), or they are unable to respond to sleep pressure. Either way, these results reveal that, in addition to changes in sleep architecture, $T$. 
A

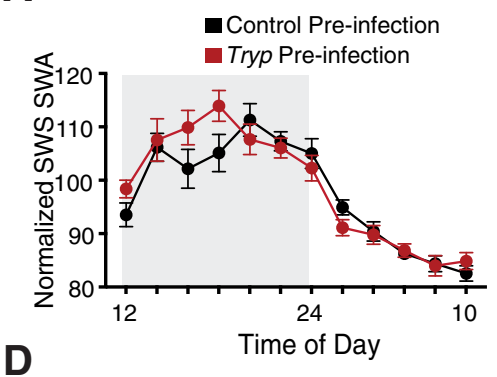

B

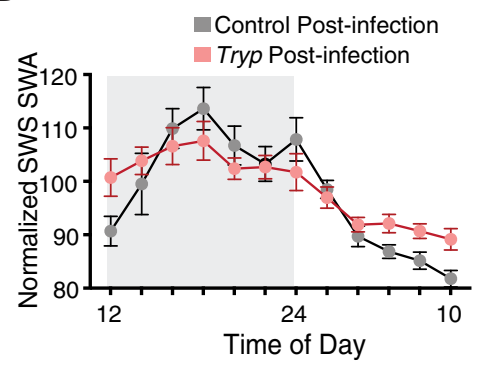

C

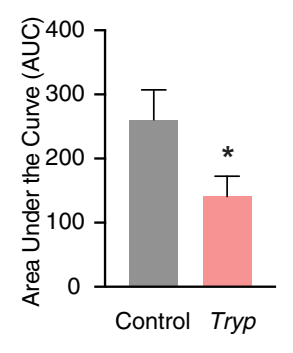

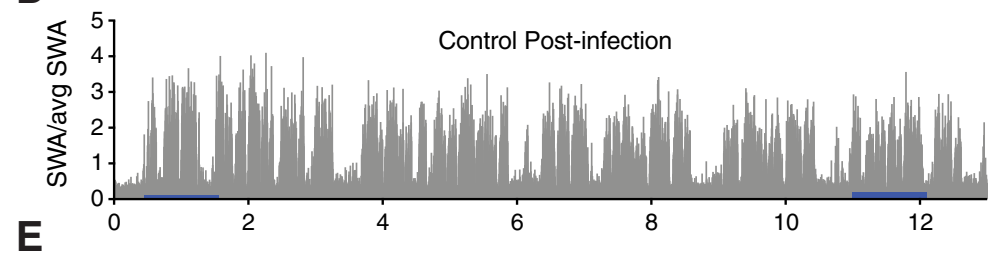

E
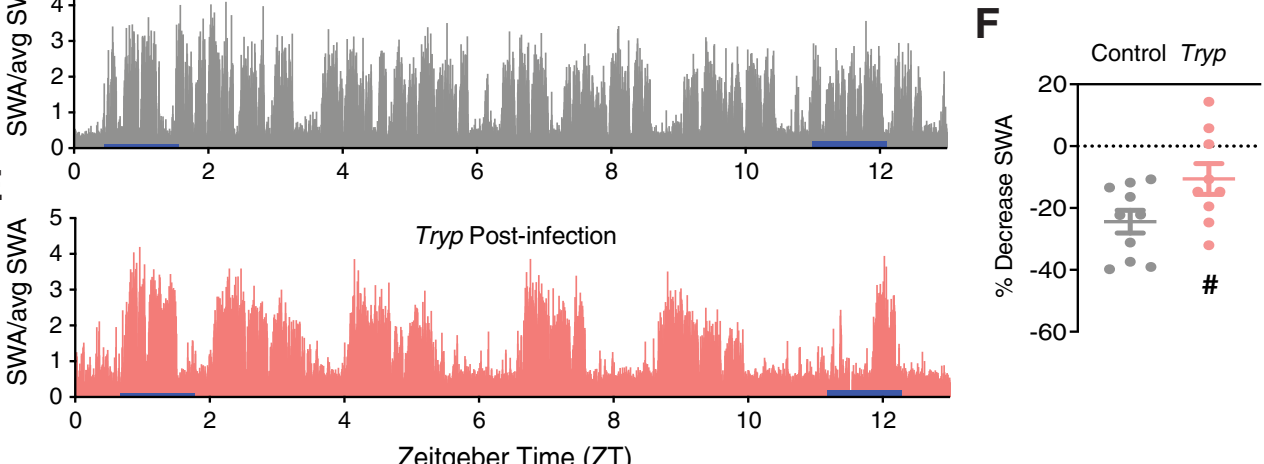

G

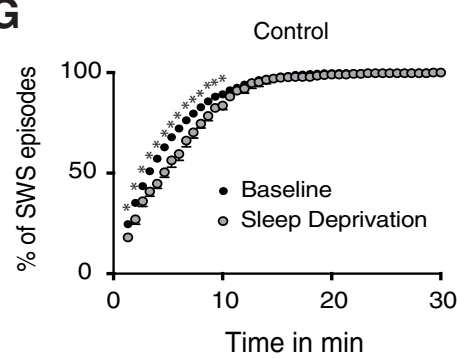

H

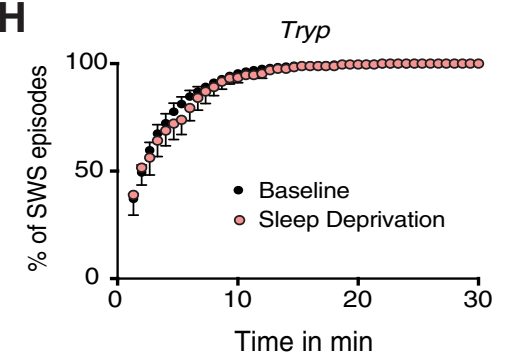

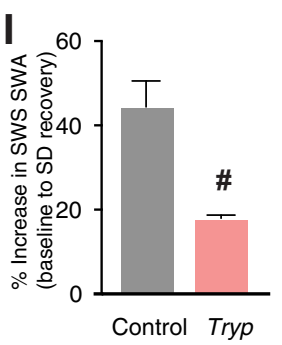

Figure 5. T. brucei infection reduces sleep need. $A$, Before infection, all animals had peak SWA during SWS in the dark phase. $B$, After infection, the peak of SWA during SWS was flattened, resulting in $(\boldsymbol{C})$ an overall decrease in the AUC for SWS SWA ( $t$ test; $\left.t_{(192)}=2.003 ; * p=0.0466\right)$. $\boldsymbol{D}$, A control mouse shows a decrease in SWA from near the beginning to the end of the light phase $(25.5 \%)$, whereas $(\boldsymbol{E})$ an infected mouse shows an attenuated SWA decrease (12\%). Blue bars indicate when measures were taken to quantify percent decrease. $\boldsymbol{F}$, Group averages of the percent decrease in SWA over the light phase show a non-significant trend toward less SWA decrease in infected mice $\left(t\right.$ test; $\left.t_{(18)}=1.962 ; \# p=0.0654\right)$. $\mathbf{G}$, When mice were exposed to a sleep deprivation protocol, control mice had a reduction in the duration of SWS episodes, indicative of consolidation (two-way ANOVA; $\left.F_{(65,650)}=6.989 ; p<0.0001\right)$. $\boldsymbol{H}, \mathrm{SWS}$ consolidation was not seen in infected animals after sleep deprivation, as the distribution of SWS episodes was not altered. $I$, Controls also had a larger rebound in the light phase than infected animals $(t$ test; $t_{(6)}=2.363 ; \# p=0.0560$ ) as determined by percent increase in SWS SWA from baseline.

brucei-infected mice have a baseline reduction in homeostatic sleep response.

Next, we evaluated sleep patterns in infected mice after sleep deprivation (Fig. 1A). Initially, we used a treadmill sleep deprivation protocol previously shown to prolong the duration of SWS episodes (SWS consolidation) and increase the SWS above baseline levels at the same circadian time (SWA rebound). Both of these parameters are indicative of increased sleep need (Bjorness et al., 2009). However, the infected mice subjected to this protocol were unable to maintain the necessary walking pace and died during the deprivation period; therefore, for the remainder of the infected mice we switched to a sleep deprivation protocol using gentle handling instead. As expected, control mice subjected to sleep deprivation showed SWS consolidation as determined by an increase in the duration of SWS episodes as a consequence of sleep need (two-way ANOVA; interaction of time and group $F_{(65,650)}=6.989, p<0.0001$; Fig. $\left.5 G\right)$. However, similar SWS consolidation was not seen in infected mice after sleep deprivation (no differences between conditions; Fig. 5H), and there was no difference in sleep achieved during the deprivation period [ $5 \mathrm{~min}$ total across controls, $6 \mathrm{~min}$ total across infected animals, $p=0.72, t$ test]. In addition, after sleep deprivation, controls had the expected SWA rebound over the light phase compared with baseline conditions, while SWA rebound was reduced in infected mice $\left(t\right.$ test, $t_{(6)}=2.363$, $\# p=0.0560$; Fig. $5 I)$. Despite these findings, there was no effect of infection on the latency to fall asleep after sleep deprivation, suggesting that there is still some sleep control, but homeostatic control is compromised (Fig. 4B). Taken together, our results suggested that mice with sleeping sickness have altered sleep homeostasis. Because the adenosine system is thought to mediate the homeostatic sleep response (Bjorness and Greene, 2009), we hypothesized that $T$. brucei infection may alter adenosine signaling.

\section{T. brucei infection alters adenosine signaling}

Activation of adenosine A1 receptors (ADORA1) is required for SWA rebound in response to loss of sleep (Bjorness et al., 2009). To investigate whether infected mice had altered adenosine signaling, we performed PPF experiments using hippocampal CA1 field recordings from control and infected mice ( $n=8$ animals/ 


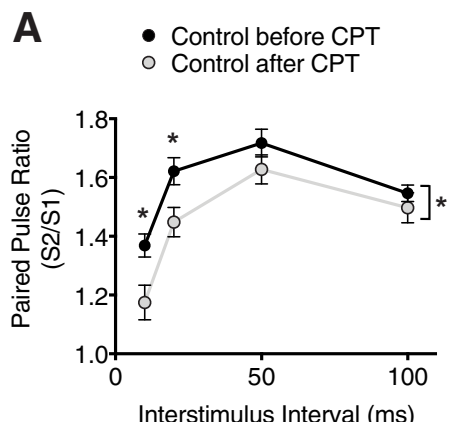

C

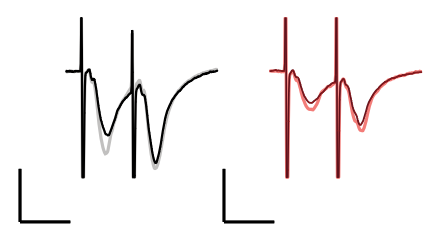

B
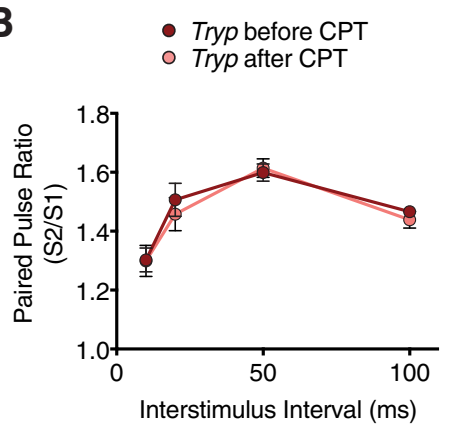

D

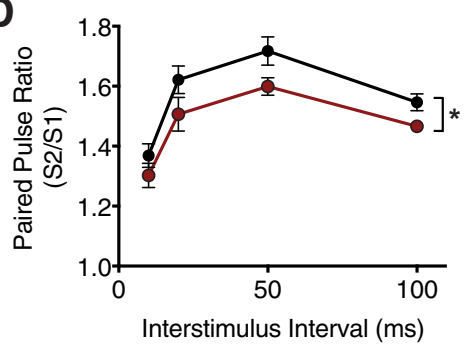

Figure 6. Hippocampal neurons from $T$. brucei-infected animals fail to respond to adenosine receptor antagonism. $\boldsymbol{A}, \boldsymbol{B}$, Paired-pulse ratios from hippocampal CA1 of control $(n=8)$ and infected $(n=8)$ animals (one slice per animal) before and after treatment with an adenosine A1 receptor antagonist, (PT. $\boldsymbol{A}$, Controls demonstrate a decrease in PPF after CPT treatment [two-way ANOVA; * main effect of treatment $F_{(1,56)}=14.41 ; p=0.0004$; * above data points indicate significant differences $(p<0.05)$ after multiple comparisons (Sidak)]. $\boldsymbol{B}$, Infected mice show no response to CPT treatment (two-way ANOVA; $F_{(1,56)}=0.3043 ; p=0.5834$ ). $C$, Representative traces from control and infected mice in $\boldsymbol{A}, \boldsymbol{B}$. D, Pre-CPT comparison between control and treated animals also reached statistical significance (two-way ANOVA; * main effect of treatment $F_{(1,56)}=11.40 ; p=0.0013$ ), suggesting that infected mice have reduced adenosine signaling. All data points are represented at mean $\pm \mathrm{SEM}$.

group), and compared paired-pulse facilitation (PPF) before and after treatment with an ADORA1 antagonist, CPT (Brundege and Dunwiddie, 1996; Dumas and Foster, 1998). Under normal conditions, extracellular adenosine acts via ADORA1 to decrease the probability of vesicular glutamate release at hippocampal Schaffer collateral synapses (presynaptic inhibition), leading to stronger PPF. Therefore, blocking these receptors with CPT should lead to attenuated PPF in mice with a functional adenosine system.

Accordingly, slices from control mice demonstrated this expected decrease in PPF after CPT treatment, indicative of basal adenosine tone (two-way ANOVA; main effect of treatment $F_{(1,56)}=14.41 ; p=0.0004$; Fig. $\left.6 A, C\right)$. However, PPF in infected mice was not changed after ADORA1 antagonist exposure (twoway ANOVA; $F_{(1,56)}=0.3043 ; p=0.5834$; Fig. $\left.6 B, C\right)$. Direct comparisons between control and infected groups shows that infected mice exhibit a reduced baseline PPF resembling the PPF reduction seen in controls after blockade. (two-way ANOVA; main effect of treatment $F_{(1,56)}=11.40 ; p=0.0013$; Fig. $\left.6 D\right)$. These findings suggest a possible reduction in tonic basal presynaptic inhibition mediated by reduced adenosine tone in infected animals.

When adenosine kinase (AdK) is overexpressed in glial cells, mice have decreased sleep drive because of decreased adenosine signaling (Palchykova et al., 2010). T. brucei infection causes activation of astrocytes (Tesoriero et al., 2018), which is also associated with increased AdK activity (Boison, 2008). Therefore, we hypothesized that infected mice may have increased expression of AdK. Alternatively, it was also possible that infected mice could have decreased adenosine tone because of decreased ADORA1 expression (Bjorness et al., 2009, 2016). To address these possibilities, we performed qRT-PCR on multiple tissues in control and infected mice. As shown in Figure $7 A$, there were no significant increases in $A d k$ expression, although there was a trend for increased expression in infected animals in frontal cortex ( $t$ test; $\left.t_{(6.02)}=2.131 ; \# p=0.0769\right)$. There was also a tendency for infected mice to have increased Adora1 mRNA expressed in frontal cortex $(t$ test; $\left.t_{(6)}=2.008 ; \# p=0.0914\right)$ and hypothalamus $\left(t\right.$ test; $\left.t_{(5.039)}=2.081 ; \# p=0.0915\right)$. Importantly, although infection increased the expression of inflammatory cytokines in multiple tissues, control mice with implanted EEG electrodes also had elevated expression of these same immune genes as compared with non-surgical controls and there were no statistical differences between groups, indicating that immune activation alone is not enough to explain the behavioral changes in infected mice (Fig. 7B). Together with the electrophysiological results, these findings suggest that T. brucei infection may alter adenosine signaling, thereby providing a possible mechanism for altered homeostatic sleep control.

\section{Discussion}

Here, we report significant alterations in sleep architecture in a mouse model of chronic sleeping sickness. Our findings recapitulate many aspects of the human sleeping sickness phenotype and provide further validation for this murine disease model. T. brucei invades the brain through the circumventricular organs (Schultzberg et al., 1988; Laperchia et al., 2016). However, treatment with suramin (Keita et al., 1997), a drug commonly used in the field to treat the first, hemolymphatic stage of this infection, is unable to penetrate the blood brain barrier. Thus, suramin treatment allows infected mice to survive systemic infection and creates a model of the second, meningeencephalitic stage of the disease. We believe that this model of sleeping sickness mimics the human infection, with parasites rarely visible in the blood circulation while infiltrating the central nervous system (Masocha and Kristensson, 2019; Tesoriero et al., 2019). Using this model, we previously found that T. brucei disrupted the circadian clock of the host, shortening the circadian behavioral period and altering clock gene expression in both central and peripheral tissues (Rijo-Ferreira et al., 2018).

In the rat, T. brucei infection alters melatonin receptor binding and neuronal activity in the master circadian pacemaker, the SCN (Kristensson et al., 1998; Lundkvist et al., 2002), and also changes clock gene expression in peripheral tissues (Lundkvist et al., 2010). Our behavioral findings are largely consistent with findings of sleep disturbances in T. brucei-infected rats (Darsaud et al., 2004; Seke Etet et al., 2012; Laperchia et al., 2017). However, our analysis was unable to detect any SOREM episodes, even when using relatively lax scoring criteria (Seke Etet et al., 2012). This may be because of differences in study conditions. Rats were typically assessed for up to $20 \mathrm{~d}$ postinfection, and therefore had systemic T. brucei parasitemia during EEG/EMG recordings, which may cause more drastic sleep alterations. In contrast, our mice received suramin treatment at $21 \mathrm{~d}$ postinfection and were recorded at $85 \mathrm{~d}$ postinfection. It is also possible that there are species differences in SOREM episodes following infection, although SOREM has been reported in mouse models of narcolepsy (Fujiki et al., 2009). Of note, the alterations we see 
A

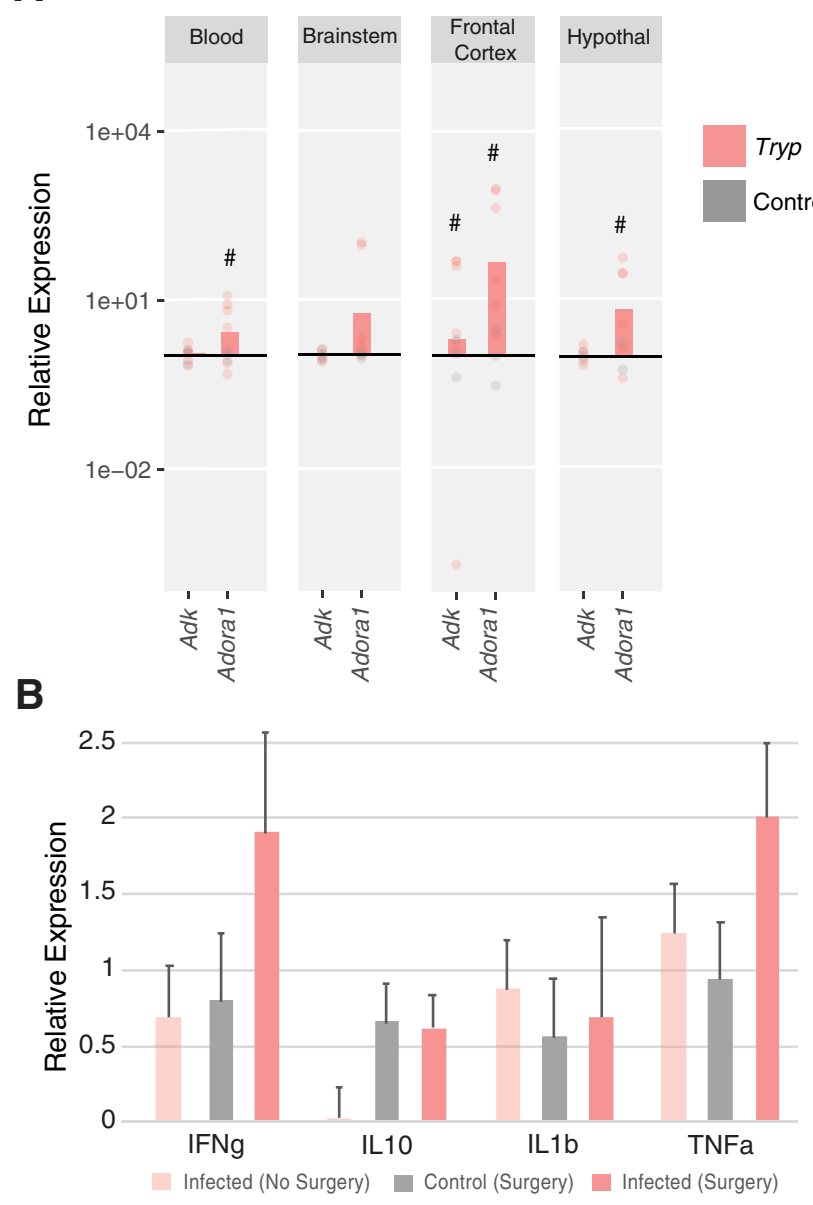

Figure 7. T. brucei infection alters adenosine A1 receptor mRNA expression in brain tissues. mRNA expression was quantified with $\mathrm{qRT}$-PCR, and data are presented for infected $(n=7)$ animals as fold-change relative to control $(n=3)$ expression. $A$, There was a trend for increased Adk expression in the frontal cortex ( $t$ test; $\left.t_{(6.02)}=2.131 ; \# p=0.0769\right)$. There were also trends for increased ADORA1 expression in infected animals in the blood ( $t$ test; $\left.t_{(6.043)}=2.196 ; \# p=0.0702\right)$, frontal cortex ( $t$ test; $\left.t_{(6)}=2.008 ; \# p=0.0914\right)$, and hypothalamus $\left(t\right.$ test; $\left.t_{(5.039)}=2.081 ; \# p=0.0915\right)$. $B$, mRNA expression of inflammatory cytokines revealed no significant differences in mice with or without surgical implantation of electrodes ( $n=3-7 /$ group). Data are presented as relative to controls without surgery.

in sleep behavior are distinct from the direct wake to REM transitions seen with narcolepsy that are mediated by the orexin system. While there is some indication that $T$. brucei infection causes orexin and $\mathrm{MCH}$ neuronal death in rats (Palomba et al., 2015) of which orexin loss would be expected to result in SOREMs, other studies have shown that only $T$. brucei gambiense induces significant damage to orexin and $\mathrm{MCH}$ neurons (Laperchia et al., 2018) as well as the SCN (Tesoriero et al., 2018) in susceptible organisms. However, these models may not fully recapitulate human sleeping sickness pathophysiology, as postmortem studies do not indicate significant neuronal damage (Kennedy, 2004).

Our findings that sleep deprivation by forced (slow) locomotion resulted in death in infected animals are intriguing. They suggest that these mice, although not enduring systemic infection at the time of testing, have either (1) decreased ability to achieve a constant level of physical activity even with slow locomotion or (2) long-lasting negative health impacts that are not otherwise apparent. In fact, studies in humans suggest that hypothalamicpituitary-adrenal (HPA) axis activity may be impacted in patients even after suramin treatment (Reincke et al., 1994). It is worth noting that the results from the modified sleep deprivation protocol used for the remaining infected animals are not straight-forward to interpret. However, this method produced similar total waking time as that of controls, and there was a similar achievement of sleep during the deprivation period with the gentle handling method as with the treadmill method, suggesting that the mice were adequately and similarly sleep deprived across groups. Nonetheless, further studies using optimized sleep deprivation procedures, possibly with cage changes and novel objects to facilitate arousal maintained, would be beneficial. It may also be useful to log the number of interventions needed to maintain wakefulness as an additional index for sleep drive. Additionally, examining the stress response in infected animals may give insights into the pervasive neuropsychiatric and clinical aspects of the meninge-encephalitic stage of sleeping sickness.

In addition to altered sleep patterns, we found that $T$. bruceiinfected mice had altered homeostatic sleep drive, both before and after sleep deprivation, suggesting the involvement of the adenosine system. Adenosine inhibits synaptic transmission in the hippocampus and basal forebrain (Arrigoni et al., 2006; Arrigoni and Rosenberg, 2006), but has been shown to activate ventrolateral preoptic (VLPO) sleep-active neurons (Morairty et al., 2004; Bjorness and Greene, 2009). Adenosine signaling mediated through the ADORA1 is necessary for SWA rebound after sleep deprivation (Bjorness et al., 2009), and increasing adenosine tone increases homeostatic sleep need (Bjorness et al., 2016). Our results suggest that $T$. brucei infection causes a decrease in baseline adenosine signaling, thereby reducing SWS SWA, indicative of the need to sleep. This may be because of decoupling of ADORA1 from intracellular signaling pathways, a hypothesis supported by the increase in Adoral mRNA in the brain, but reduced response to ADORA1 antagonism shown with in vitro electrophysiology (PPF). It would be interesting in future studies to examine ADORA1 signaling pathways in neurons from infected mice.

Interestingly, adenosine release from astrocytes, as well as neurons, modulates sleep pressure (Halassa et al., 2009), and lipopolysaccharide (LPS) injections have been shown to increase the astrocytic release of adenosine to increase sleep drive (Nadjar et al., 2013). T. brucei has also been shown to activate astrocytes in mice (Hunter et al., 1992), but it is unclear whether T. brucei infection directly induces adenosine release. We used an indirect measure of adenosine metabolism (i.e., quantifying AdK mRNA) and did not detect a significant increase across tissues. However, these findings from bulk tissues are by no means conclusive and may warrant further investigation using single-cell resolution to identify the genes that are activated and suppressed by T. brucei infection.

T. brucei secrete prostaglandins (Kubata et al., 2000; Kristensson et al., 2010), which causes the release of inflammatory cytokines, many of which act as somnogens (Krueger et al., 2001; Bryant et al., 2004; Imeri and Opp, 2009). More specifically, systemic infections, LPS injection, or administration of the cytokines TNF-a or IL-1B all increase SWS while reducing REM sleep (Morrow and Opp, 2005; Krueger et al., 2011; Tesoriero et al., 2019), particularly with severe inflammation (Majde and Krueger, 2005). In contrast with these studies, we show that $T$. brucei-infected mice do not sleep more. Our in-depth analysis of sleep architecture suggests that, although infected mice attempt to enter deep sleep, disruption of SWS may decrease the number of successful REM episodes. Moreover, although infected mice show an increase in the expression of inflammatory cytokines, this is not above the levels of controls receiving surgery. Thus, 
we speculate that the main influence of T. brucei on sleep, at least in this advanced stage of infection, is not merely via cytokine signaling. Alternatively, it is possible that T. brucei can both promote and combat cytokine signaling (Lundkvist et al., 2004), including regulating the expression of suppressors of cytokine signaling (SOCS; Alexander, 2002). Finally, it is possible that the reduced homeostatic sleep drive seen here is a consequence of a decrease in ability to achieve sleep. It would be informative for future studies to determine whether T. brucei infection prevents increased sleep following pharmacological or environmental interventions (for example, the dual orexin receptor antagonist suvorexant (Winrow et al., 2011) or a mild temperature increase (Szymusiak and Satinoff, 1981; Roussel et al., 1984).

\section{References}

Alexander WS (2002) Suppressors of cytokine signalling (SOCS) in the immune system. Nat Rev Immunol 2:410-416.

Arrigoni E, Rosenberg PA (2006) Nitric oxide-induced adenosine inhibition of hippocampal synaptic transmission depends on adenosine kinase inhibition and is cyclic GMP independent. Eur J Neurosci 24:2471-2480.

Arrigoni E, Chamberlin NL, Saper CB, McCarley RW (2006) Adenosine inhibits basal forebrain cholinergic and noncholinergic neurons in vitro. Neuroscience 140:403-413.

Bjorness TE, Greene RW (2009) Adenosine and sleep. Curr Neuropharmacol 7:238-245.

Bjorness TE, Kelly CL, Gao T, Poffenberger V, Greene RW (2009) Control and function of the homeostatic sleep response by adenosine A1 receptors. J Neurosci 29:1267-1276.

Bjorness TE, Dale N, Mettlach G, Sonneborn A, Sahin B, Fienberg AA, Yanagisawa M, Bibb JA, Greene RW (2016) An adenosine-mediated glial-neuronal circuit for homeostatic sleep. J Neurosci 36:3709-3721.

Blum J, Schmid C, Burri C (2006) Clinical aspects of 2541 patients with second stage human African trypanosomiasis. Acta Trop 97:55-64.

Boison D (2008) The adenosine kinase hypothesis of epileptogenesis. Prog Neurobiol 84:249-262.

Borbély AA, Daan S, Wirz-Justice A, Deboer T (2016) The two-process model of sleep regulation: a reappraisal. J Sleep Res 25:131-143.

Bringmann H (2018) Sleep-active neurons: conserved motors of sleep. Genetics 208:1279-1289.

Brundege JM, Dunwiddie TV (1996) Modulation of excitatory synaptic transmission by adenosine released from single hippocampal pyramidal neurons. J Neurosci 16:5603-5612.

Bryant PA, Trinder J, Curtis N (2004) Sick and tired: does sleep have a vital role in the immune system? Nat Rev Immunol 4:457-467.

Buguet A, Bert J, Tapie P, Tabaraud F, Doua F, Lonsdorfer J, Bogui P, Dumas M (1993) Sleep-wake cycle in human African trypanosomiasis. J Clin Neurophysiol 10:190-196.

Buguet A, Bourdon L, Bouteille B, Cespuglio R, Vincendeau P, Radomski MW, Dumas M (2001) The duality of sleeping sickness: focusing on sleep. Sleep Med Rev 5:139-153.

Buguet A, Bisser S, Josenando T, Chapotot F, Cespuglio R (2005) Sleep structure: a new diagnostic tool for stage determination in sleeping sickness. Acta Trop 93:107-117.

Caljon G, Van Reet N, De Trez C, Vermeersch M, Pérez-Morga D, Van Den Abbeele J (2016) The dermis as a delivery site of Trypanosoma brucei for tsetse flies. PLoS Pathog 12:e1005744.

Capewell P, Cren-Travaillé C, Marchesi F, Johnston P, Clucas C, Benson RA, Gorman TA, Calvo-Alvarez E, Crouzols A, Jouvion G, Jamonneau V, Weir W, Stevenson ML, O'Neill K, Cooper A, Swar NK, Bucheton B, Ngoyi DM, Garside P, Rotureau B, et al. (2016) The skin is a significant but overlooked anatomical reservoir for vector-borne African trypanosomes. Elife 5:e17716.

Chen L, Thakkar MM, Winston S, Bolortuya Y, Basheer R, McCarley RW (2006) REM sleep changes in rats induced by siRNA-mediated orexin knockdown. Eur J Neurosci 24:2039-2048.

Dantz B, Edgar DM, Dement WC (1994) Circadian rhythms in narcolepsy: studies on a 90 minute day. Electroencephalogr Clin Neurophysiol 90:24-35.

Darsaud A, Bourdon L, Mercier S, Chapotot F, Bouteille B, Cespuglio R, Buguet A (2004) Twenty-four-hour disruption of the sleep-wake cycle and sleep-onset REM-like episodes in a rat model of African trypanosomiasis. Sleep 27:42-46.

Dumas TC, Foster TC (1998) Late developmental changes in the ability of adenosine Al receptors to regulate synaptic transmission in the hippocampus. Brain Res Dev Brain Res 105:137-139.

Engstler M, Boshart M (2004) Cold shock and regulation of surface protein trafficking convey sensitization to inducers of stage differentiation in Trypanosoma brucei. Genes Dev 18:2798-2811.

Franco JR, Simarro PP, Diarra A, Jannin JG (2014) Epidemiology of human African trypanosomiasis. Clin Epidemiol 6:257-275.

Franken P, Chollet D, Tafti M (2001) The homeostatic regulation of sleep need is under genetic control. J Neurosci 21:2610-2621.

Fujiki N, Cheng T, Yoshino F, Nishino S (2009) Specificity of direct transition from wake to REM sleep in orexin/ataxin-3 transgenic narcoleptic mice. Exp Neurol 217:46-54.

Halassa MM, Florian C, Fellin T, Munoz JR, Lee SY, Abel T, Haydon PG, Frank MG (2009) Astrocytic modulation of sleep homeostasis and cognitive consequences of sleep loss. Neuron 61:213-219.

Hunter CA, Jennings FW, Kennedy PG, Murray M (1992) Astrocyte activation correlates with cytokine production in central nervous system of Trypanosoma brucei brucei-infected mice. Lab Invest 67:635-642.

Imeri L, Opp MR (2009) How (and why) the immune system makes us sleep. Nat Rev Neurosci 10:199-210.

Jafari B, Mohsenin V (2010) Polysomnography. Clin Chest Med 31:287-297.

Keita M, Bouteille B, Enanga B, Vallat JM, Dumas M (1997) Trypanosoma brucei brucei: a long-term model of human African trypanosomiasis in mice, meningo-encephalitis, astrocytosis, and neurological disorders. Exp Parasitol 85:183-192.

Kennedy PG (2004) Human African trypanosomiasis of the CNS: current issues and challenges. J Clin Invest 113:496-504.

Kristensson K, Claustrat B, Mhlanga JD, Møller M (1998) African trypanosomiasis in the rat alters melatonin secretion and melatonin receptor binding in the suprachiasmatic nucleus. Brain Res Bull 47:265-269.

Kristensson K, Nygård M, Bertini G, Bentivoglio M (2010) African trypanosome infections of the nervous system: parasite entry and effects on sleep and synaptic functions. Prog Neurobiol 91:152-171.

Krueger JM, Opp MR (2016) Sleep and microbes. Int Rev Neurobiol 131:207-225.

Krueger JM, Obál FJ, Fang J, Kubota T, Taishi P (2001) The role of cytokines in physiological sleep regulation. Ann NY Acad Sci 933:211-221.

Krueger JM, Clinton JM, Winters BD, Zielinski MR, Taishi P, Jewett KA, Davis CJ (2011) Involvement of cytokines in slow wave sleep. Prog Brain Res 193:39-47.

Kubata BK, Duszenko M, Kabututu Z, Rawer M, Szallies A, Fujimori K, Inui T, Nozaki T, Yamashita K, Horii T, Urade Y, Hayaishi O (2000) Identification of a novel prostaglandin $\mathrm{f}(2 \mathrm{alpha})$ synthase in Trypanosoma brucei. J Exp Med 192:1327-1338.

Laperchia C, Palomba M, Seke Etet PF, Rodgers J, Bradley B, Montague P, Grassi-Zucconi G, Kennedy PG, Bentivoglio M (2016) Trypanosoma brucei invasion and T-cell infiltration of the brain parenchyma in experimental sleeping sickness: timing and correlation with functional changes. PLoS Negl Trop Dis 10:e0005242.

Laperchia C, Tesoriero C, Seke-Etet PF, La Verde V, Colavito V, GrassiZucconi G, Rodgers J, Montague P, Kennedy PGE, Bentivoglio M (2017) Expression of interferon-inducible chemokines and sleep/wake changes during early encephalitis in experimental African trypanosomiasis. PLoS Negl Trop Dis 11:e0005854.

Laperchia C, Xu YZ, Mumba Ngoyi D, Cotrufo T, Bentivoglio M (2018) Neural damage in experimental Trypanosoma brucei gambiense infection: hypothalamic peptidergic sleep and wake-regulatory neurons. Front Neuroanat 12:13.

Lazarus M, Oishi Y, Bjorness TE, Greene RW (2019) Gating and the need for sleep: dissociable effects of adenosine A1 and A2A receptors. Front Neurosci 13:740.

Lundkvist GB, Hill RH, Kristensson K (2002) Disruption of circadian rhythms in synaptic activity of the suprachiasmatic nuclei by African trypanosomes and cytokines. Neurobiol Dis 11:20-27.

Lundkvist GB, Kristensson K, Bentivoglio M (2004) Why trypanosomes cause sleeping sickness. Physiology (Bethesda) 19:198-206.

Lundkvist GB, Sellix MT, Nygård M, Davis E, Straume M, Kristensson K, Block GD (2010) Clock gene expression during chronic inflammation 
induced by infection with Trypanosoma brucei brucei in rats. J Biol Rhythms 25:92-102.

Majde JA, Krueger JM (2005) Links between the innate immune system and sleep. J Allergy Clin Immunol 116:1188-1198.

Masocha W, Kristensson K (2019) Human African trypanosomiasis: how do the parasites enter and cause dysfunctions of the nervous system in murine models? Brain Res Bull 145:18-29.

Morairty S, Rainnie D, McCarley R, Greene R (2004) Disinhibition of ventrolateral preoptic area sleep-active neurons by adenosine: a new mechanism for sleep promotion. Neuroscience 123:451-457.

Morrow JD, Opp MR (2005) Diurnal variation of lipopolysaccharide-induced alterations in sleep and body temperature of interleukin-6-deficient mice. Brain Behav Immun 19:40-51.

Nadjar A, Blutstein T, Aubert A, Laye S, Haydon PG (2013) Astrocytederived adenosine modulates increased sleep pressure during inflammatory response. Glia 61:724-731.

Nelson AB, Faraguna U, Zoltan JT, Tononi G, Cirelli C (2013) Sleep patterns and homeostatic mechanisms in adolescent mice. Brain Sci 3:318-343.

Nishino S, Mignot E (1997) Pharmacological aspects of human and canine narcolepsy. Prog Neurobiol 52:27-78.

Palchykova S, Winsky-Sommerer R, Shen HY, Boison D, Gerling A, Tobler I (2010) Manipulation of adenosine kinase affects sleep regulation in mice. J Neurosci 30:13157-13165.

Palomba M, Seke-Etet PF, Laperchia C, Tiberio L, Xu YZ, Colavito V, GrassiZucconi G, Bentivoglio M (2015) Alterations of orexinergic and melaninconcentrating hormone neurons in experimental sleeping sickness. Neuroscience 290:185-195.

Porkka-Heiskanen T, Strecker RE, Thakkar M, Bjorkum AA, Greene RW, McCarley RW (1997) Adenosine: a mediator of the sleep-inducing effects of prolonged wakefulness. Science 276:1265-1268.

Reincke M, Heppner C, Petzke F, Allolio B, Arlt W, Mbulamberi D, Siekmann L, Vollmer D, Winkelmann W, Chrousos GP (1994) Impairment of adrenocortical function associated with increased plasma tumor necrosis factor-alpha and interleukin-6 concentrations in African trypanosomiasis. Neuroimmunomodulation 1:14-22.

Rijo-Ferreira F, Carvalho T, Afonso C, Sanches-Vaz M, Costa RM, Figueiredo LM, Takahashi JS (2018) Sleeping sickness is a circadian disorder. Nat Commun 9:62.
Rijo-Ferreira F, Takahashi JS (2020) Sleeping Sickness: a tale of two clocks. Frontiers Cellular and Infection Microbiology. 10:551.

Roussel B, Turrillot P, Kitahama K (1984) Effect of ambient temperature on the sleep-waking cycle in two strains of mice. Brain Res 294:67-73.

Saper CB, Scammell TE, Lu J (2005) Hypothalamic regulation of sleep and circadian rhythms. Nature 437:1257-1263.

Schultzberg M, Ambatsis M, Samuelsson EB, Kristensson K, van Meirvenne N (1988) Spread of Trypanosoma brucei to the nervous system: early attack on circumventricular organs and sensory ganglia. J Neurosci Res 21:56-61.

Seke Etet PF, Palomba M, Colavito V, Grassi-Zucconi G, Bentivoglio M, Bertini G (2012) Sleep and rhythm changes at the time of Trypanosoma brucei invasion of the brain parenchyma in the rat. Chronobiol Int 29:469-481.

Szymusiak R, Satinoff E (1981) Maximal REM sleep time defines a narrower thermoneutral zone than does minimal metabolic rate. Physiol Behav 26:687-690.

Takeuchi T, Duszkiewicz AJ, Sonneborn A, Spooner PA, Yamasaki M, Watanabe M, Smith CC, Fernández G, Deisseroth K, Greene RW, Morris RG (2016) Locus coeruleus and dopaminergic consolidation of everyday memory. Nature 537:357-362.

Tesoriero C, Xu YZ, Mumba Ngoyi D, Bentivoglio M (2018) Neural damage in experimental Trypanosoma brucei gambiense infection: the suprachiasmatic nucleus. Front Neuroanat 12:6.

Tesoriero C, Del Gallo F, Bentivoglio M (2019) Sleep and brain infections. Brain Res Bull 145:59-74.

Toda H, Williams JA, Gulledge M, Sehgal A (2019) A sleep-inducing gene, nemuri, links sleep and immune function in Drosophila. Science 363:509-515.

Trindade S, Rijo-Ferreira F, Carvalho T, Pinto-Neves D, Guegan F, ArestaBranco F, Bento F, Young SA, Pinto A, Van Den Abbeele J, Ribeiro RM, Dias S, Smith TK, Figueiredo LM (2016) Trypanosoma brucei parasites occupy and functionally adapt to the adipose tissue in mice. Cell Host Microbe 19:837-848.

Winrow CJ, Gotter AL, Cox CD, Doran SM, Tannenbaum PL, Breslin MJ, Garson SL, Fox SV, Harrell CM, Stevens J, Reiss DR, Cui D, Coleman PJ, Renger JJ (2011) Promotion of sleep by suvorexant-a novel dual orexin receptor antagonist. J Neurogenet 25:52-61. 